http://dx.doi.org/10.1590/0370-44672018720181

\begin{abstract}
Alex Miranda ${ }^{1,2}$
https://orcid.org/0000-0001-8606-7705

Beck Nader ${ }^{1,3}$

https://orcid.org/0000-0002-7215-7379
\end{abstract}

${ }^{1}$ Universidade Federal de Minas Gerais - UFMG,

Escola de Engenharia, Departamento de Engenharia de Minas, Belo Horizonte - Minas Gerais - Brasil.

E-mails: ${ }^{2}$ alexflavio21@ufmg.br, ${ }^{3}$ beckn@demin.ufmg.br, beckn@usp.br

\title{
Miningo
}

\section{Direct sequencing of blocks in stochastic models with multi- mines and multi-destinations}

\begin{abstract}
Mining Scheduling is the one that maximizes profit from mining over time. By means of computational methods, the deposit is discretized in blocks and algorithms are used to consummate this objective. The methods that are widely known nowadays for mining scheduling optimization of a discrete block model were based on graph theory, and among those most used by the mining industry is a solution found by Lerchs and Grossmann (1965) which, together with other methods, was consolidated as the process of traditional mining planning. For both supply and multi-destination models, there is a limitation of the current methodology, since it consists of the optimization of each mine separately which may not be a global optimization solution. This article proposes an optimization of the benefit in a stochastic model through the DBS (Direct Block Scheduling) for a copper mining complex with two mines, a pre-existing copper stockpile and two treatment streams, comparing several scenarios and analyzing the best alternative for the proposed problem.
\end{abstract}

keywords: mining planning, direct block sequencing, mining.

\section{Introduction}

Linear programming techniques for mine scheduling were pioneered by Johson (1968). Johson developed a mathematical model to solve the problem of long-term programming using the Dantzig-Wolfe - originally developed by American mathematicians George Dantzig and Phil Wolfe - (1960) principle that uses the algorithm for the decomposition of the complex master problem into subproblems. The computational time for processing made this method impossible. The algorithm of Lerchs and Grossmann (1965), for having its computational implementation performed by Whittle (1993), has been used by various mining planning packages ever since. The process of scheduling the mining process involves the removal of at least two types of materials: ore and waste. The mining problem, then, can be modeled in terms of entire programming (HOCHBAUM e CHEN, 2000). The typical formulation of this model is as follows: $\bullet b \in B$ : set of all B blocks.

- $t \in T$ : set of periods in a time frame $T$.

- $v_{b t}$ : value associated with extraction of the block $\boldsymbol{b}$ in period t.

- $c_{b}$ : resource consumption associated with block extraction $\boldsymbol{b}$ (ton).

- $\underline{C}, \bar{C}$ : minimum, maximum resource tied to any period (ton).

- $y_{b t}: 1$ if block $\boldsymbol{b}$ is extracted in period $\boldsymbol{t}, 0$ otherwise (variable). 


$$
\operatorname{Max} \sum_{b \in B} \sum_{t \in T} v_{b t} y_{b t}
$$

subject

$$
\begin{gathered}
\sum_{t \in T} y_{b t} \leq 1 \forall b, \\
\underline{C} \leq \sum_{b \in B} c_{b} y_{b t} \leq \bar{C} \forall t, \\
y_{b t} \leq \sum_{T=1}^{t} y_{b t} \forall b, b^{\prime} \in B_{b}, t, \\
y_{b t} \in\{0,1\} \forall b, t .
\end{gathered}
$$

The illustrated method is a simple example of modeling, and it is possible to include stockpiles, blending, etc. Over the years several algorithms have been developed to solve the problem of the mining sequencing. Gershon (1983) and Barbaro and Ramani (1986) have proposed mixed integer programming (MIP) to optimize long-term production scheduling models. Seymour (1994) and Tolwinski (1998) presented approaches based on dynamic programming methods for optimizing the production sequence using the maximum

\section{Surface based mine scheduling}

A mine sequencing can be understood as a set of surfaces in the space that divides the orebody into parts to be extracted during different periods (ALMEIDA, 2013). This surfacebased approach facilitates the management of slopes, improving efficiency

This function is a variation on the model of Ramazan and Dimitrakopoulos (2012)unlike equation (1), there is inserted the term $S$ referring to the number of simulations of the mineral body, $\boldsymbol{s}$ : index of each simulation, $s=1,2, \ldots, S$. $T$ is the number of periods that also defines

\section{Materials and methods}

SimSched software was used as a tool to optimize the sequencing. As for the manipulation of the models and construction of the benefit function, the

\subsection{Block Model}

The block model of mine 01 (Figure 1) was regularized, since it was composed of irregular blocks. After its net present value as the optimization criterion. Ravenscroft (1992) suggested a new approach when proposing that production scheduling should be planned in conditionally simulated alternative models to verify the sensitivity of the production schedule in relation to the uncertainty of the contents. Smith and Dimitrakopoulos (1999) proposed an approach in which several optimal sequencings are obtained, one for each simulated model.

Ramazan and Dimitrakopoulos (2012) propose a general formulation to

in SIP solution formulations because it is not necessary to do precedence of block extraction.

The centroids of the blocks become part of a network of measured points of a topographic surface and can therefore be represented by a binary

$\operatorname{Max} \frac{1}{S} \sum_{S=1}^{S} \sum_{t=1}^{T} \sum_{c=1}^{M} \sum_{z=1}^{Z} V_{c, t, s}^{z}\left(X_{c, t}^{z}-X_{c, t-1}^{z}\right)$

the number of surfaces to be considered; $\boldsymbol{t}$ : index of periods, $t=1,2, \ldots, T . M$ is the amount of cells on each surface, where $\boldsymbol{M}=\boldsymbol{x} . \boldsymbol{y}$ represents the total of blocks in both $x$ and $y$ dimensions; $c$ : location index of each cell / block, $c=1,2, \ldots, M . Z$ is the number of levels in the orebody model;

graphic software Studio OP (Datamine) was used. The block models were granted by Datamine containing oxide and sulfide ore with $40 \mathrm{Cu}$ grade simulations, but

regularization, the two block models were merged in order to perform the study with a single model and a field was optimize the mining production program by means of a fixed-stages StochasticInteger Programming (SIP) model that successfully optimizes open-pit mine scheduling under geological uncertainty.

In this research, the general objective is the application of a methodology for the sequencing of mining with simulated models, using computational tools based on surface mining to solve the problem, evaluating the influence of some variables (such as the inclusion or not of stockpiles).

variable $\boldsymbol{x}_{c t}^{2}=\mathbf{1}$ if mining in period $t$ and 0 , otherwise. A fundamental aspect of this approach is the need to associate blocks with surface cells and to make comparisons of their elevations in space. The formulation of this model is detailed below:

z: level index, $z=1,2, \ldots, Z$. $\boldsymbol{V}^{z}$ its is the accumulated discounted value of the block (c, $z$ ) and of all the blocks above the scenario $\mathrm{s}$ and period $t . \boldsymbol{x}_{c, t}^{z}$ is the binary variable that assumes value 1 if the block $(c, z)$ is the last block being drawn in period $t$ over $c$ and 0 (zero) otherwise.

to reduce the processing time of the two mines, only $15 \mathrm{Cu}$ grade simulations were used (randomly chosen only to illustrate the case study).

created to distinguish one mine from the other (MINA field).

Mine 02 (Figure 2) consists of oxide 
and sulfide ore, but with estimates of only the oxide ore grade. Below are pre-

Figure 1

Detail of the "box" of the mine 01 block model with a vertical cut (Cu grade).

Figure 2

Detail of the "box" of the mine 02 block model with a vertical cut (Cu grade).

\subsection{Benefit Function}

The benefit function was elaborated according to parameters determined on

Table 1

Economic parameters used in the model.

For each $\mathrm{Cu}$ grade simulation, there was calculated the mass recovery in each

Table 2

Mass recovery according Cu grade. sented the $2 \mathrm{D}$ and $3 \mathrm{D}$ visualization of the model with copper grade for this mine.
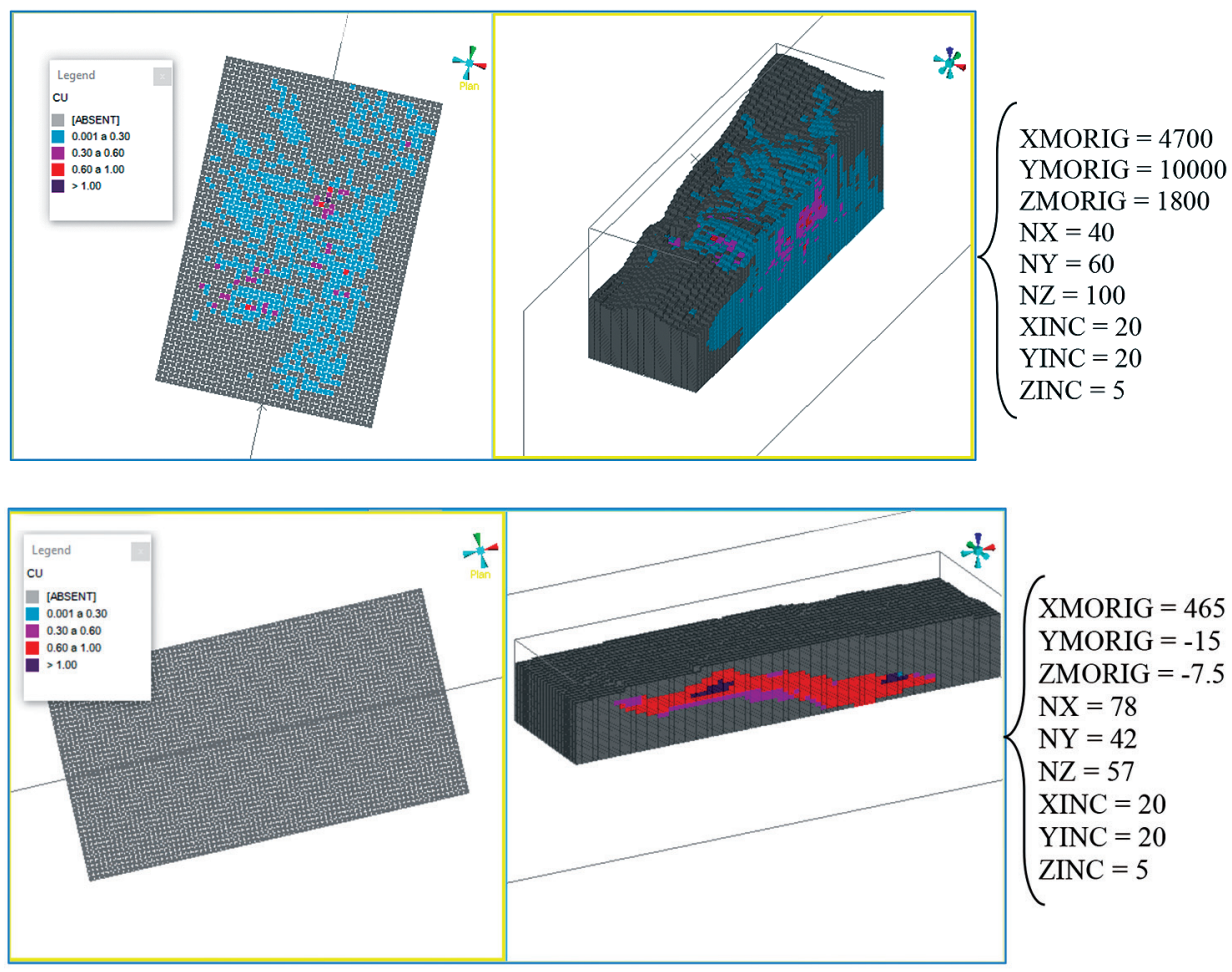

the basis of some feasibility studies done the values used to make the value of each by the author himself. The Table 1 with

Economic Parameters

\begin{tabular}{c|c|c}
\hline & Value & Unit \\
\hline Copper price & 1.7 & US\$/lb \\
\hline Mining cost & 1.95 & US\$/t \\
\hline Rehandling cost $^{*}$ & 0.05 & US\$/t \\
\hline Flotation cost & 3.5 & US\$/t \\
\hline Leach cost & 3.4 & US\$/t \\
\hline Selling cost & 10.0 & US\$/t
\end{tabular}

treatment circuit (or route) considered according to the Table 2 below:

Mass recovery by process

\begin{tabular}{c|c|c}
\hline \multicolumn{3}{|c|}{ Mass recovery by process } \\
\hline Range grade $(\mathrm{Cu})$ & Value & Unit \\
\hline $0>\% \mathrm{Cu}<0.2999$ & $\mathrm{R}=2.33 \times \% \mathrm{Cu}$ & $\mathrm{R}=2.16 \times \% \mathrm{Cu}$ \\
\hline $0.3>\% \mathrm{Cu}<0.5999$ & 0.70 & 0.65 \\
\hline$\% \mathrm{Cu}>0.6$ & 0.82 & 0.65 \\
\hline
\end{tabular}

\subsection{Configuring multiple destinations and scenarios}

As defined a priori, the treatment process consists of two destinations corresponding to the two possible processes for concentrating the R.O.M mass from the mines. Three (3) scenarios were considered for comparing NPV values in each alternative. The characteristics of each alternative are:

- Scenario 01: Direct feed of ore in each plant, whereby the oxide ore can go to the flotation or leach circuits and the sulfide ore can only go to the leach circuit;

- Scenario 02: Same as scenario 01, but the sulfide can be stored in a stockpile for later feeding.

- Scenario 03: It considers a pile of pre-existing oxide ore (with a certain capacity and only $\mathrm{Cu}$ grade average) that can feed the plant directly at any time of the mining sequence. The flowchart of this scenario is presented (Figure 3): 


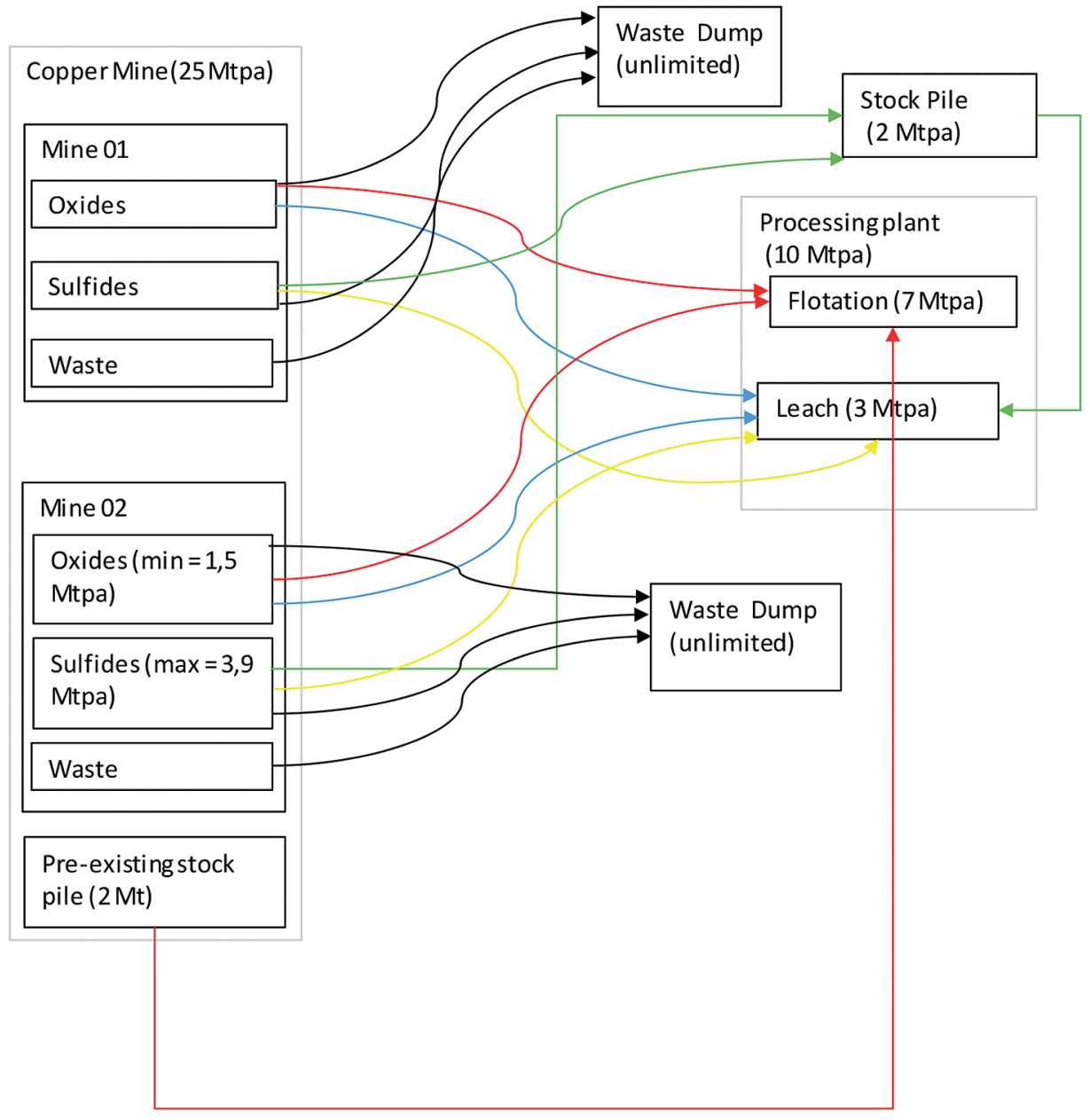

Figure 3

Simplified Scenario 03 Flowchart.

\section{Results}

For scenario 01, 170.8 Mt of ore (oxide and sulfide) was mined and the accumulated strip ratio was $1.65 \mathrm{t} / \mathrm{t}$ and the useful life of mine reached 19 years. For scenario 02 (Figure 5), approximately $175.4 \mathrm{Mt}$ of ore was mined, and the accumulated strip ratio was $1.70 \mathrm{t} / \mathrm{t}$, and also the useful life of mine was 19 years. In scenario 03 (Figure 6), $179 \mathrm{Mt}$ of ore was mined with an accumulated strip ratio of $1.71 \mathrm{t} / \mathrm{t}$. With the strategy of having an initial stockpile, the

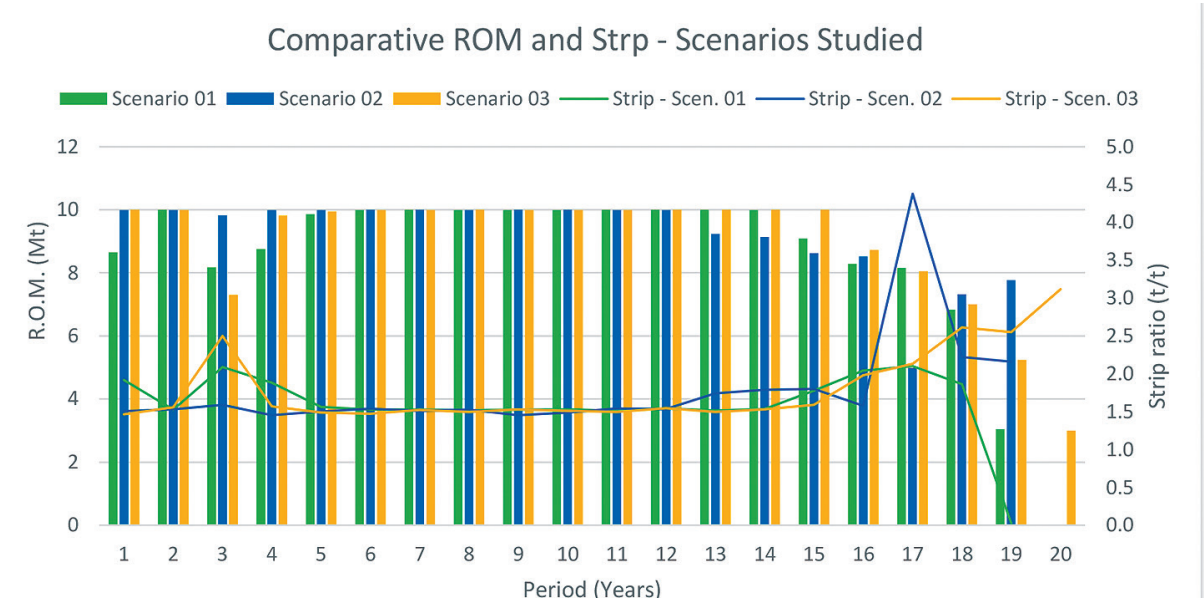

\section{Discussion}

Each scenario attempted to meet the need for the subsequent scenario. After evaluating each strategy, the NPV variations of all scenarios were compared, and it was concluded that the most favorable scenario from the financial return point of view would be scenario 02 (using the stock pile - Table total life of mine was 20 years. However, there were already $2 \mathrm{Mt}$ of ore available, therefore, only $177 \mathrm{Mt}$ were mined in the mine. A summary chart of each scenario comparing the R.O.M. and the strip ratio of each scenario is presented (Figure 4).
Figure 4

Comparison between the studied scenarios.

3 and Figure 5). The table with the NPV and the variation of the minimum and maximum value in each alternative is presented below: 


\begin{tabular}{|c|c|c|c|c|}
\hline \multirow[b]{2}{*}{ Scenario } & \multicolumn{3}{|c|}{ Accumulated NPV ( US\$ $\times 10^{6}$ ) } & \multirow[b]{2}{*}{$\% \Delta(\max -\min )$} \\
\hline & Min & Max & Expected & \\
\hline 01 & $86,615.3$ & $104,354.0$ & $91,466.0$ & $20 \%$ \\
\hline 02 & $100,374.0$ & $120,123.0$ & $105,292.0$ & $20 \%$ \\
\hline 03 & $92,630.4$ & $110,955.0$ & $97,626.0$ & $20 \%$ \\
\hline
\end{tabular}

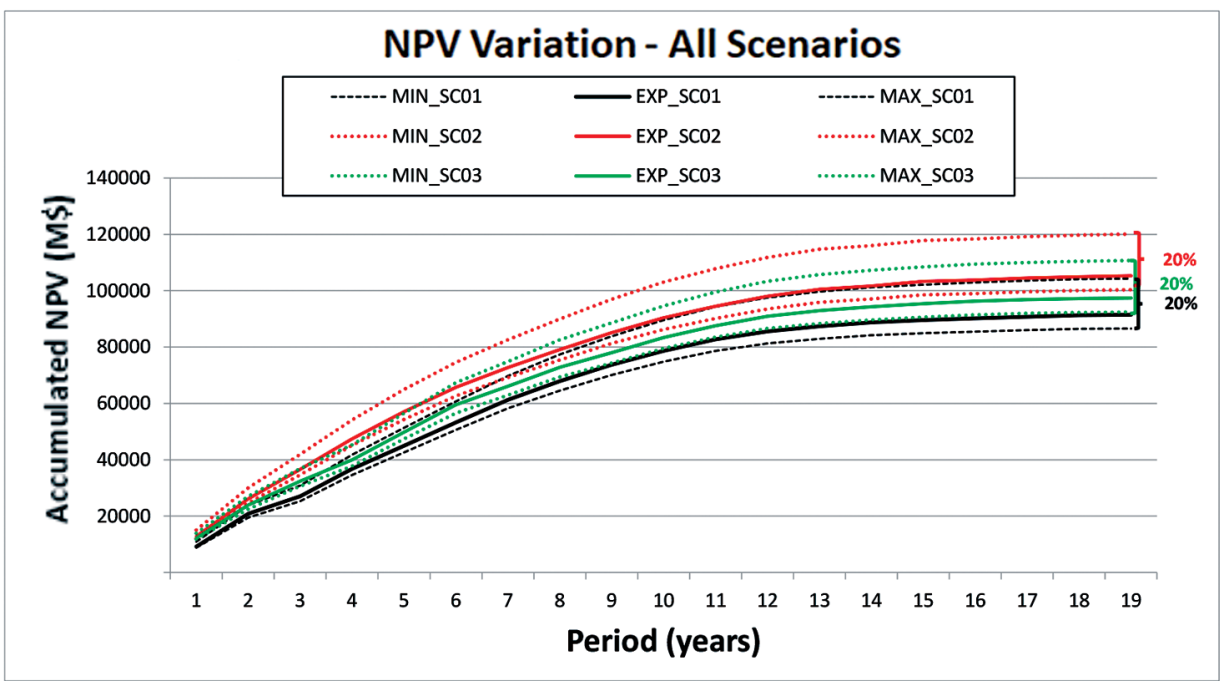

For scenario 01 (Figure 6), the traditional sequencing was done considering the average of the 15 simulations (E-type) and the use of the algorithm of Lerchs and Grossmann (LG). The chart below (Figure 6), the traditional sequencing was done considering the average of the 15 simulations (E-type) and the use of the algorithm of Lerchs and Grossmann (LG). The chart below shows that there is a difference of $11 \%$ between the NPV values accumulated until the end of the

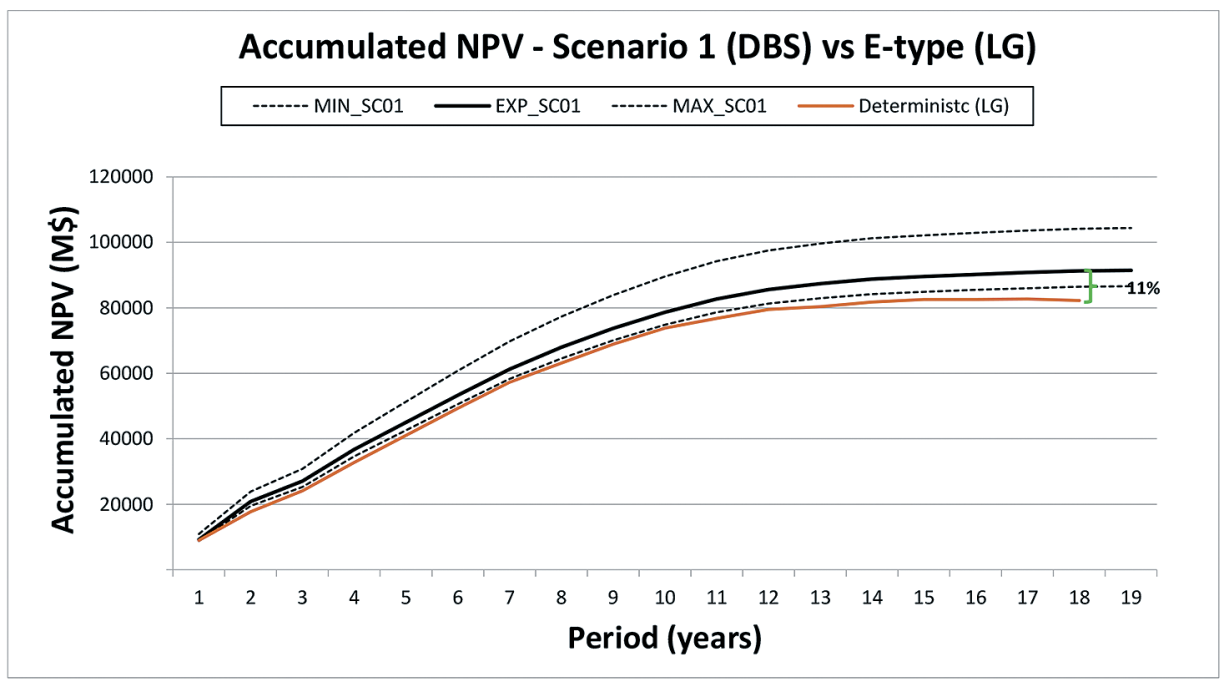

\section{Conclusions}

It was observed that DBS is an excellent tool to solve the problem of mine sequencing with multiple simulation grades and that, in addition to working with these simulations, it was shown that it has the ability to process different scenarios with lower and higher complexity degrees. Among the main advantages of the approach with multiple models and multiple destinations that currently are imposed on mining projects, we can highlight:
- Obtaining the results in a single step of work, without the need to first find the best economic envelope and sectorization of pit in portions and then perform the mine sequencing;

- With the currently existing computer tools, you can do multiple analyzes with different inputs in different scenarios and SimSched software was robust enough to generate these scenarios;

- Analogous to packages that work
Table 3

NPV values (with their

variations) for each studied scenario.

Figure 5

Comparison of NPV

variation in different scenarios.

18th period (last year sequencing by traditional methodology). shows that there is a difference of $11 \%$ between the NPV values accumulated until the end of the 18th period (last year sequencing by traditional methodology).

Figure 6

Comparison of NPV

between scenario 01 and E-type (LG).

with deterministic models, it is also possible to work with stockpiles and maximize the project value;

- In the software used (SimSched) it was also possible to include some operational restrictions such as vertical rate advance and minimum width for mining and bottom, which makes the solution closer to the operational reality of mining;

- As the operational details and blending restrictions are incorporated into 
the sequencing, the most intense is the delay in finding the solution.

Despite the advantages mentioned above, there is still the drawback with the processing time for more demanding processes in terms of complexity and the need for the models to be composed of blocks with the same size so that they can be processed together. Given that the studied scenarios were able to reach their objectives (without exceeding the capacity limit of the mine and feeding the plant's feed flows), it was observed that scenario 02 - Figure 5 (strategy of using stockpiles) managed to obtain a higher economic return expected among the three studied scenarios. However, the ranges of variation in each scenario allow to affirm that, with small adaptations in supply (geological model) and demand (metal price) policies, it will lead to a variety of alternative scenarios and opportunities for greater gains that can be exploited in future implementations through sensitivity analysis.

\section{References}

ALMEIDA, A. Surface constrained stochastic life-of-mine production scheduling. Montreal: MCGill University, 2013. p. 191.

BARBARO, R. W., RAMANI, R. V. Generalized multiperiod MIP model for production scheduling and processing facilities selection and location. Mining Engineering, p. 107-114, February 1986.

DANTZIG, G. B., WOLFE, P. Decomposition principle for linear programs. Operations Research 8. p. 101-111, 1960.

GERSHON, M. E. Mine scheduling optimization with mixed integer programming. Mining Engineering, p. 351-354, April 1983.

HOCHBAUM, D. S., CHEN, A. Performance analysis and best implementations of old and new algorithms for the open-pit mining problem. p. 894-914, 2000.

JOHNSON, T. B. Optimum Open Pit Mine Production Scheduling. Berkeley, 1968. p. 120.

LERCHS, H., GROSSMANN, L. Optimum design of open-pit mine. Montreal: 1965. p. 17-24.

RAMAZAN, S. D., DIMITRAKOPOULOS, R. Production scheduling with uncertain supply: a new solution to the open pit mining problem. Optimization and Engineering, 2012.

SMITH, M., DIMITRAKOPOULOS, R. Influence of deposit modelling on mine production scheduling. [S.1.], p. 173-178. 1999.

SEYMOUR, F. Finding the mining sequence and cutoff-grade schedule that maximizes net present value. 1994. p. 1880-1884.

TOLWINSKI, B. Scheduling production for open pit mines. London: 1998. p. 651-662.

WHITTLE, J. Four-D Whittle Open Pit Opimisation Software. Melbourne: Whittle Programming Pty Ltd, 1993. (User Manual).

Received: 10 December 2018 - Accepted: 16 July 2019. 